\title{
Bone loss patterns in cortical, subcortical, and \\ trabecular compartments during simulated \\ microgravity
}

Tomas Cervinka, Harri Sievänen, Jari Hyttinen and Jörn Rittweger

J Appl Physiol 117:80-88, 2014. First published 8 May 2014; doi:10.1152/japplphysiol.00021.2014

You might find this additional info useful...

This article cites 35 articles, 3 of which can be accessed free at:

/content/117/1/80.full.html\#ref-list-1

Updated information and services including high resolution figures, can be found at:

/content/117/1/80.full.html

Additional material and information about Journal of Applied Physiology can be found at:

http://www.the-aps.org/publications/jappl

This information is current as of August 11, 2014.

Journal of Applied Physiology publishes original papers that deal with diverse areas of research in applied physiology, especially those papers emphasizing adaptive and integrative mechanisms. It is published 12 times a year (monthly) by the American

Physiological Society, 9650 Rockville Pike, Bethesda MD 20814-3991. Copyright (C) 2014 by the American Physiological Society.

ISSN: 0363-6143, ESSN: 1522-1563. Visit our website at http://www.the-aps.org/. 


\title{
Bone loss patterns in cortical, subcortical, and trabecular compartments during simulated microgravity
}

\author{
Tomas Cervinka, ${ }^{1,2}$ Harri Sievänen, ${ }^{3}$ Jari Hyttinen, ${ }^{1,2}$ and Jörn Rittweger ${ }^{4,5}$ \\ ${ }^{1}$ Department of Electronics and Communications Engineering, Tampere University of Technology, Tampere, Finland; \\ ${ }^{2}$ Institute of Bioscience and Medical Technology, Tampere, Finland; ${ }^{3}$ Bone Research Group, UKK Institute, Tampere, \\ Finland; ${ }^{4}$ Institute of Aerospace Medicine, German Aerospace Center, Cologne, Germany; and ${ }^{5}$ Institute for Biomedical \\ Research into Human Movement and Health, Manchester Metropolitan University, Manchester, United Kingdom
}

Submitted 13 January 2014; accepted in final form 5 May 2014

Cervinka T, Sievänen H, Hyttinen J, Rittweger J. Bone loss patterns in cortical, subcortical, and trabecular compartments during simulated microgravity. J Appl Physiol 117: 80-88, 2014. First published May 8, 2014; doi:10.1152/japplphysiol.00021.2014.—Disuse studies provide a useful model for bone adaptation. A direct comparison of these studies is, however, complicated by the different settings used for bone analysis. Through pooling and reanalysis of bone data from previous disuse studies, we determined bone loss and recovery in cortical, subcortical, and trabecular compartments and evaluated whether the study design modulated skeletal adaptation. Peripheral quantitative tomographic (pQCT) images from control groups of four disuse studies with a duration of 24, 35, 56, and 90 days were reanalyzed using a robust threshold-free segmentation algorithm. The pQCT data were available from 27 young healthy men at baseline, and at specified intervals over disuse and reambulation phases. The mean maximum absolute bone loss (mean $\pm 95 \% \mathrm{CI}$ ) was $6.1 \pm 4.5 \mathrm{mg} / \mathrm{mm}$ in cortical, $2.4 \pm 1.6 \mathrm{mg} / \mathrm{mm}$ in subcortical, and $9.8 \pm 9.1 \mathrm{mg} / \mathrm{mm}$ in trabecular compartments, after 90 days of bed rest. The percentage changes in all bone compartments were, however, similar. During the first few weeks after onset of reambulation, the bone loss rate was systematically greater in the cortical than in the trabecular compartment $(P<0.002)$, and this was observed in all studies except for the longest study. We conclude that disuse-induced bone losses follow similar patterns irrespective of study design, and the largest mean absolute bone loss occurs in the cortical compartment, but apparently only during the first 60 days. With longer study duration, trabecular loss may become more prominent.

bed rest; immobilization; osteoporosis; pQCT; image processing

BONE LOSS, RESULTING IN REDUCED whole bone strength, is triggered by several factors such as aging, hormonal disturbances, disease, disuse, or microgravity during space flight. Therefore, the study of processes in bone tissue as responses to various conditions or treatments that could prevent bone deterioration is one of the main goals of clinical bone research.

Over the past two decades, bed rest studies have been used to investigate skeletal responses to reduced loading, disuse, or simulated microgravity (15). The first bed rest studies used planar dual-energy X-ray absorptiometry (DXA) imaging (1, $14,24,28,30,35,36)$, but this method is known to be compromised by inherent inaccuracy due to the violation of the two-component assumption (homogeneous soft tissue and bone) and by the inability to yield reliable information on actual bone geometry and structure $(6,26)$. Recently, several studies have used peripheral quantitative computed tomogra-

Address for reprint requests and other correspondence: T. Cervinka, Finnmedi 1, 4th Floor, Biokatu 6, 33520 Tampere, Finland (e-mail: tomas.cervinka @tut.fi). phy (pQCT) $(4,18,21-23,29,33)$ or its high-resolution version (3). In contrast to DXA, pQCT provides a reliable assessment of bone cross-sectional geometry and allows separation into trabecular and cortical compartments (25).

The pQCT-based bed rest studies primarily used manufacturer-proposed thresholding procedures for bone analysis, and results have therefore not been reported in the same way. At the distal tibia site, some studies reported changes in total bone mineral content (BMC) (21) or cortical and trabecular BMC $(22,29)$ from baseline data collection until the onset of reambulation. Other studies reported changes in cortical and trabecular BMC $(18,23)$ or total and trabecular bone mineral density (BMD) $(4,33)$ at various time points during bed rest and subsequent reambulation. As a result, the direct between-study comparison of results is complicated. Simple threshold-based approaches can also result in a considerable variation in the cross-sectional areas of bone compartments $(2,11,12)$ and further compromise bone analysis in a longitudinal setting. Therefore, we hypothesized that a robust analysis of the same subject-specific cortical, subcortical, and trabecular bone crosssectional areas of all consecutive pQCT images throughout the entire longitudinal study would reveal compartment-specific changes in skeletal responses that have remained masked in conventional thresholding approaches. Moreover, the pooling of the largest set of available data obtained from four out of seven disuse studies performed with pQCT so far, and reanalyzing the image data in a similar robust way, is expected to provide more accurate insight into bone adaptation patterns with higher statistical power.

The studies from which the pQCT data were obtained were the 90-day Long Term Bed Rest (LTBR) study, carried out in Toulouse, France, in 2001 and 2002 (21); the 56-day first Berlin Bed Rest (BBR) study, carried out in Berlin, Germany, in 2003 and 2004 (18); the 24-day Unilateral Lower Limb Suspension (ULLS) study, carried out in Alsager, U.K., in 2005 (23); and the 35-day Valdoltra study, carried out in Ankaran, Slovenia, in 2007 (22).

The primary objective of the present study was to carry out a commensurate analysis of pQCT-measured cortical, subcortical, and trabecular bone mineral content (BMCc, BMCsub, $\mathrm{BMCt}$, respectively) at the distal tibia by applying a robust, recently developed, threshold-free segmentation algorithm (8) on the same bone areas using data pooled from the abovementioned four prospective disuse trials each with a different duration and design (18, 21-23). More specifically, we assessed 1) whether this analysis could confirm the notion that the majority of bone loss occurs in the cortical compartment (1, $18,20,22)$ as opposed to the trabecular compartment, which 
Table 1. Characteristics of the four disuse studies

\begin{tabular}{|c|c|c|c|c|c|c|}
\hline Study & Place & Year & Duration, days & Position & Legs immobilized & Countermeasures \\
\hline LTBR & Toulouse, France & $2001 / 2002$ & 90 & $-6^{\circ}$ & 2 & Flywheel exercise, pamidronate \\
\hline BBR & Berlin, Germany & $2003 / 2004$ & 56 & $0^{\circ}$ & 2 & Resistive vibration exercise \\
\hline Valdoltra & Ankaran, Slovenia & 2007 & 35 & $0^{\circ}$ & 2 & \\
\hline ULLS & Alsager, UK & 2005 & 24 & $+90^{\circ}$ & 1 & \\
\hline
\end{tabular}

LTBR, Long Term Bed Rest; BBR, Berlin Bed Rest; ULLS, Unilateral Lower Limb Suspension.

seems to be affected in patients with spinal cord injury or in astronauts during long space flights $(27,32)$; and 2) whether the different study designs modulated the bone loss and recovery patterns.

\section{MATERIALS AND METHODS}

Study procedures. In this study, the pQCT data were reanalyzed from the control groups only (i.e., the subjects who underwent disuse only) of the LTBR study $(n=9)$, the first BBR study $(n=10)$, the Valdoltra study $(n=10)$, and the ULLS study $(n=8)$. The protocols of these four studies are described elsewhere in detail $(18,21-23)$ and summarized in Table 1. The ethical committee at each facility approved the study protocols, and each participant (all were male) in the study gave written informed consent before the study began (18, 21-23).

In short, LTBR study participants were randomly divided into three groups in which the influence of a one-time treatment by pamidronate prior to bed rest and a flying wheel exercise during bed rest, both used as counter measures to bone loss, was investigated and compared with results from the control group (strict bed rest only). BBR study participants were randomly divided into control and resistive vibration exercise groups. All Valdoltra study participants underwent strict bed rest only. This study was designed primarily to investigate the differences in changes in cortical and trabecular bone compartments and to determine whether cortical bone loss exceeded trabecular bone loss during immobilization. All ULLS study participants underwent a right leg suspension. This was achieved with a $7.5-\mathrm{cm}$ soled shoe on the left foot, and participant stability was achieved with bilateral crutches. For the present study, the left leg data of control participants in the LTBR and BBR studies and the right leg data of all participants of the Valdoltra and ULLS studies were used. The anthropometric characteristics of these groups are shown in Table 2.

Bone measurements. In all four studies, pQCT (XCT 2000; Stratec Medizintechnik, Pforzheim, Germany) was performed in the same way according to a previously published imaging protocol (19). In short, pQCT images of the distal tibia of the left leg (LTBR study), the right leg (Valdoltra and ULLS studies), and both legs (BBR study) were obtained at the $4 \%$ distal site of the tibia. The pixel size of the pQCT image was $0.4 \times 0.4 \mathrm{~mm}$ in the LTBR study and $0.5 \times 0.5 \mathrm{~mm}$ in the BBR, Valdoltra, and ULLS studies. The translational scan speed was $30 \mathrm{~mm} / \mathrm{s}$, and the slice thickness was $2.5 \mathrm{~mm}$ in all studies.

In the LTBR study, measurements were obtained 14 and 7 days prior to the start of bed rest $(B R-14$ and $B R-7$ were considered as baseline measurements), and on days $28,44,57,69$, and 89 during the bed rest phase $(\mathrm{BR}+28, \mathrm{BR}+44, \mathrm{BR}+57, \mathrm{BR}+69$, and $\mathrm{BR}+89$, respectively), and on days $14,90,180$, and 360 after reambulation ( $R+14, R+90, R+180$, and $R+360$, respectively). In the BBR study, measurements were obtained 3 days prior and 2 days after the start of the bed rest phase $(\mathrm{BR}-3$ and $\mathrm{BR}+2$ baseline measurements); on days $17,31,45$, and 55 during the bed rest phase $(\mathrm{BR}+17, \mathrm{BR}+31$, $\mathrm{BR}+45$, and $\mathrm{BR}+55$, respectively); and on days $4,14,28,90,180$, and 360 after reambulation $(\mathrm{R}+4, \mathrm{R}+14, \mathrm{R}+28, \mathrm{R}+90, \mathrm{R}+180$, and $\mathrm{R}+360$, respectively). In the Valdoltra study, measurements were obtained 2 days prior to bed rest and in the morning of the first day of bed rest phase ( $\mathrm{BR}-2$ and $\mathrm{BR}+1$ baseline measurements), on the last day of bed rest phase $(B R+35)$, and 14 days after reambulation $(\mathrm{R}+14)$. In the ULLS study, measurements were obtained 9 days and 1 day prior to unilateral suspension (ULS -9 and ULS -1 baseline measurements); on days 7, 14, and 21 during the unilateral suspension (ULS + 7, ULS +14, and ULS +21, respectively); and on days 4, 9, 35, and 90 after reambulation $(\mathrm{R}+4, \mathrm{R}+9, \mathrm{R}+35$, and $\mathrm{R}+90$, respectively).

Data processing and analysis. For each participant, the raw data from the first baseline pQCT image was used to determine an individual reference template of the cortical area $(\mathrm{CoA})$ that was applied to all follow-up images of the given participant to determine changes in cortical, subcortical, and trabecular bone mass within the same cross-sectional area through the entire disuse and reambulation periods. In short, an accurate threshold-free segmentation algorithm recently developed by Cervinka et al. (8) was used to determine the CoA of the baseline image. Next, the reference CoA template was created by adding a 1-pixel-thick layer periosteally and a 2-pixel-thick layer endocortically to the detected CoA to compensate for any small differences in bone alignment between the baseline image and follow-up images. Then, the reference CoA template was anatomically aligned with every follow-up image by employing a fast, rigid image registration implemented in Slicer 3D version 3.6 (available at http:// www.slicer.org) (16). Thereafter, two peeling thresholds, $180 \mathrm{mg} / \mathrm{cm}^{3}$ (outer border) and $480 \mathrm{mg} / \mathrm{cm}^{3}$ (inner border), were applied to the CoA to exclude soft tissues and bone tissue pixels that apparently did not represent actual cortical bone. After this peeling procedure, the subcortical area (SubA) was defined as the immediate 4-pixel-wide region inside the $\mathrm{CoA}$, and the trabecular area $(\operatorname{TrA})$ was defined as the area inside the SubA. A flow chart of the image processing procedures is depicted in Fig. 1 and an example of BMD distribution within the detected CoA is shown in Fig. 2.

Table 2. Anthropometric characteristics of the study subjects

\begin{tabular}{|c|c|c|c|c|c|c|}
\hline Study & Group & Number of subjects & Age, years & Height, $\mathrm{cm}$ & Weight, kg & BMI, $\mathrm{kg} / \mathrm{m}^{2}$ \\
\hline LTBR & Control & 8 & $31.3(3.3)^{\boldsymbol{U}, \boldsymbol{V}}$ & $173.4(3.2)^{\boldsymbol{B}}$ & $73.0(3.8)^{\mathrm{b}}$ & $24.3(0.9)$ \\
\hline BBR & Control & 10 & $33.4(6.6)^{U, V}$ & $184.5(7.0)^{L}$ & $79.9(8.0)^{1}$ & $23.4(1.3)$ \\
\hline Valdoltra & Control & 10 & $23.9(2.5)^{B, L, U}$ & $179.7(7.7)$ & $77.3(11.0)$ & $23.9(2.8)$ \\
\hline ULLS & Control & 8 & $19.0(0.8)^{\mathrm{B}, \boldsymbol{L}, \mathrm{V}}$ & $179.3(5.0)$ & $72.0(9.1)$ & $22.4(2.6)$ \\
\hline$P$ for variance & & - & 0.005 & 0.13 & 0.32 & 0.36 \\
\hline$P$ for means & & - & $<0.001$ & $<0.001$ & 0.03 & 0.42 \\
\hline
\end{tabular}

Columns for age, height, weight, and body mass index (BMI) are means and (variances). The bottom row contains $P$ value differences across studies; the row above it contains $P$ values for homogeneity of variance. Superscript letters $L, B, V$, and $U$ denote differences from the LTBR, BBR, Valdoltra, and ULLS studies, respectively, with $P<0.05$ for small letters, $P<0.01$ for capital letters, and $P<0.001$ for bold italic capital letters. See Table 1 for abbreviations. 


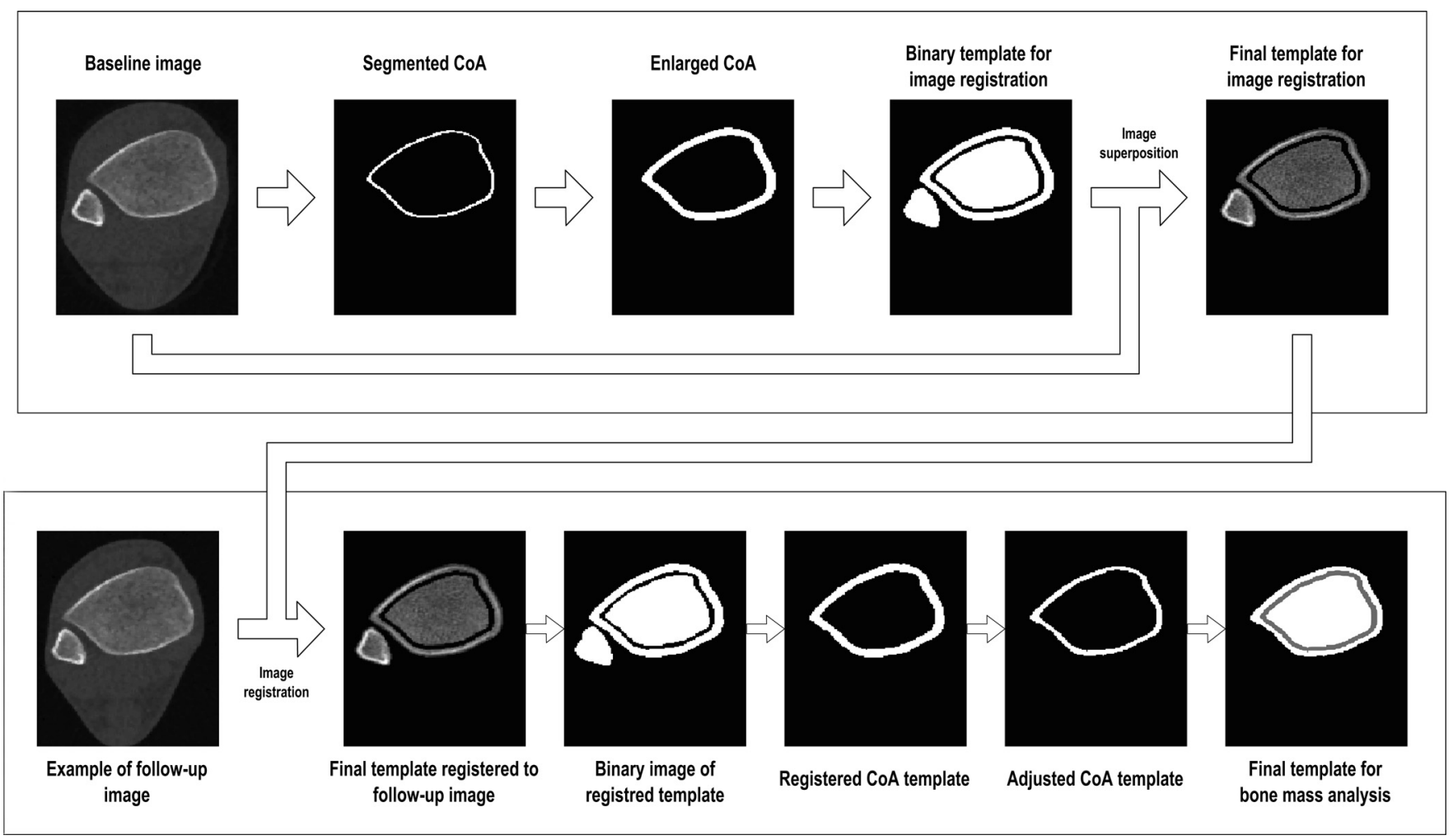

Fig. 1. Example of image processing procedure for cortical area (CoA), subcortical area (SubA), and trabecular area (TrA) determination. First, the CoA, in baseline image, was determined by analysis on the basis of a statistical approach and novel segmentation method developed previously by Cervinka et al. (7, 8). Second, the CoA template obtained from baseline data was created on the basis of segmented CoA enlarged by 2 pixels along the periosteal border and by 2 pixels along the endosteal border. This enlargement was carried out to compensate for small, expected variations in the total bone cross-sectional area that arose from positioning errors between the reference (the first baseline) image and all follow-up images. Third, the baseline CoA template was fitted to all follow-up images by using a fast, rigid registration algorithm implemented in Slicer 3D ver. 3.6. Fourth, registered CoA templates were adjusted using the superposition of the original data (processed by median filtering with a $3 \times 3$ window) into the templates. This adjustment was performed to compensate the previous enlargement procedure by removing pixels along the endosteal border that clearly contain nonbone tissues (peeling with threshold $180 \mathrm{mg} / \mathrm{mm}^{3}$ ) and along the periosteal border contour that contains trabecular bone (peeling 1-pixel-thick contour with retaining pixels whose density was above threshold set to 480 $\mathrm{mg} / \mathrm{mm}^{3}$ ). The adjusted CoA templates were considered as CoAs for the following cortical bone mass analysis. Fifth, the SubA was defined as a 4-pixel-wide border zone of area enclosed by the CoAs and TrA, as the area enclosed by SubA.

Data sets from two participants in the LTBR study and one participant in the ULLS study had to be excluded because of a failed image registration process and an inability to perform image registration within acceptable limits (the cortical compartments of the fol- low-up data did not fit into the reference CoA template due to apparent bone misalignment). Because of similar image registration problems with data from the left leg in three BBR study participants and because longitudinal data sets from the right leg were available,
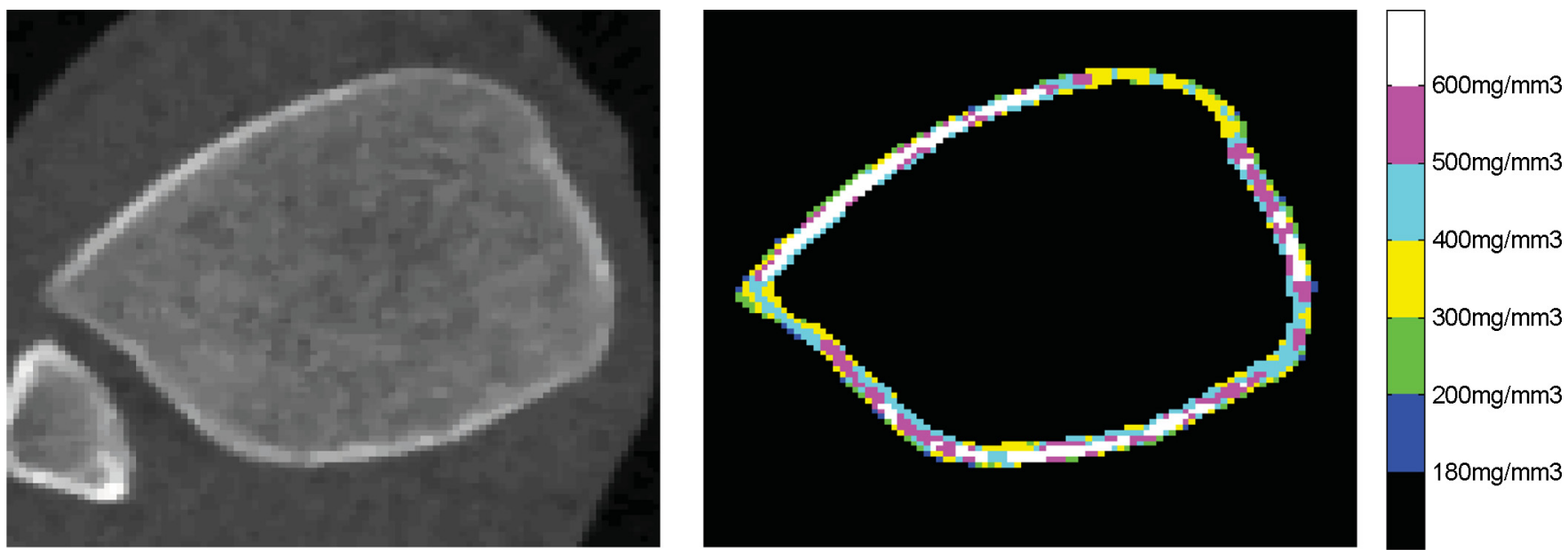

Fig. 2. Example of detected CoA: original peripheral quantitative computed tomographic image (left), and corresponding detected CoA with marked bone mineral density distribution $(r i g h t)$. 
Table 3. Baseline bone mineral content values for cortical, subcortical, and trabecular compartments of all disuse studies

\begin{tabular}{lcccc}
\hline \hline Study & LTBR & BBR & Valdoltra & ULLS \\
\hline BMCc & $132.3(20.3)$ & $141.7(23.8)$ & $146.3(29.2)$ & $134.0(18.4)$ \\
BMCsub & $56.7(6.7)$ & $66.4(8.1)$ & $71.5(8.9)$ & $71.0(9.1)$ \\
BMCt & $221.0(28.8)$ & $205.9(24.9)$ & $218.8(31.1)$ & $226.1(41.8)$ \\
\hline
\end{tabular}

Values are means and (SD) in $\mathrm{mg} / \mathrm{mm}$. BMCc, cortical bone mineral content; BMCsub, subcortical bone mineral content; BMCt, trabecular bone mineral content.

the three data sets from the left leg were replaced with right leg data. Furthermore, to avoid any inaccuracy caused by substantial bone misalignment during the follow-up, data from the follow-up images were also excluded when the CoA differed more than $10 \%$ from the baseline CoA (5 images from the LTBR study, 3 images from the BBR study, 3 images from the Valdoltra study, and 8 images from the ULLS study). The proportion of excluded images out of all the 348 images was $5.5 \%$, which was considered satisfactory.

Because of the generally limited number of participants in the disuse studies and for the sake of retaining statistical power, we imputed values for the missing or excluded data points. The imputed values were estimated by linear interpolation using data from two neighboring time points. Note that imputation was not performed for the last follow-up image if it was missing (i.e., two images in the BBR study, and one image in the ULLS study).

Statistical analyses. Anthropometric data are given as means and standard deviation (SD). ANOVA was used to assess whether any significant differences existed across the studies in terms of age, height, weight, or body mass index (BMI). The level for statistical significance was set to $\alpha=0.05$. Further, mean values and $95 \%$ confidence intervals $(95 \% \mathrm{CI})$ of BMC are given as descriptive statistics, if not otherwise indicated. Given the small number of participants in the disuse studies, no formal statistical testing was performed, but the $95 \%$ CI was considered adequate to indicate within-group changes and to compare between-group changes during the disuse and subsequent reambulatory periods. Changes in BMC during the disuse and reambulation periods were expressed as mean absolute (in $\mathrm{mg} / \mathrm{mm}$ ) and relative (in \%) changes from baseline (Table 3). The baseline value was specifically defined as the mean of the two baseline data points described above for each study (see section Bone measurements). These two data points also provided relevant information on the short-term error of BMC measurements and facilitated an appropriate interpretation of the changes in BMC (Table 4). Using the two baseline measurements, the short-term error (in \%) was calculated as follows:

$$
E r_{s h}=\frac{100}{N} \sum \frac{\left|\bar{x}_{i, B L}-x_{i, B L 1}\right|}{\bar{x}_{i, B L}}
$$

where $\bar{x}_{i, B L}$ is the mean baseline value of $i$-th subject, $\bar{x}_{i, B L 1}$ is the value of the first baseline measurement of $i$-th subject, and $N$ is the total number of participants.

A two-sample $t$-test with assumption of unequal variances was performed to assess whether the bone loss at the end of immobilization (EIBL), the maximal postreambulatory bone loss (PRABL $_{\max }$, present at day $\mathrm{R}+14$ for bed-rest studies and $\mathrm{R}+35$ for the ULLS study) in relation to EIBL, as well as their rates [defined as the slope of the tangent in the above-mentioned time points (bone loss/day)] were higher in the cortical than in the trabecular compartment. Multipleregression analysis was performed to assess the influence of the disuse duration and baseline BMC values on EIBL, PRABL, and their rates.

Furthermore, to characterize the time courses of the mean absolute changes in BMC during disuse, we used simple quadratic and linear models and an exponential model proposed by Fyhrie and Schaffler (10). The formulation of these models is the following:

$$
\begin{gathered}
\text { quadratic model : } \quad B M C_{l}(t)=a t^{2}+b t \\
\text { linear model: } \quad B M C_{l}(t)=b t+c
\end{gathered}
$$

exponential model : $\quad B M C_{l}(t)=0.7 B M C_{\text {baseline }}\left(1-e^{-b t}\right)$

where $B M C_{l}$ is the absolute change from the baseline at time $t, a$ and $b$ are time constants describing the rate of change in $\mathrm{BMC}$, and $c$ is an intercept coefficient of the linear model. It was assumed that the biological minimum is $30 \%$ of the baseline BMC (10). Therefore, $0.7 \mathrm{BMC}_{\text {baseline }}$ represents the theoretical maximal bone loss. Of note, pooled data from all three bed rest studies was used for modeling (the ULLS study was excluded because of its different study design), with the exception of the baseline value in the linear model. The goodness of fit of different models in explaining changes in BMC was compared by an $F$-test.

The time course of recovery in BMC during reambulation from minimum BMC values was analyzed with the modified exponential model for the LTBR and BBR studies only because the Valdoltra study did not include data after 14 days of reambulation. In Equation 4 , the $0.7 \mathrm{BMC}_{\text {baseline value was replaced by the mean PRABL }}$ max of the given study. The time course of the reambulatory changes in BMC was analyzed only for the LTBR and BBR studies because the data sets from these studies comprised sufficiently long 1-yr follow-up data. Because these studies had different durations of disuse, the follow-up data had to be analyzed separately. The level of significance was set at 0.05 for all the above-mentioned tests.

\section{RESULTS}

Anthropometric data. Table 2 displays participants' descriptive data in different disuse studies. Participants in the ULLS study were the youngest, followed by the Valdoltra participants, and the participants from the LTBR and BBR studies. Height was also different across the studies $(P<0.001)$, with BBR subjects being taller than LTBR subjects $(P=0.003)$. BBR subjects were also heavier than LTBR subjects $(P=$ $0.032)$, but BMI was comparable across the studies $(P=0.42)$.

Bone loss patterns during disuse. Absolute and percentage changes in BMCc, BMCsub, and BMCt at the distal tibia during disuse are shown in Fig. 3 and Fig. 4, respectively. For bed rest studies, mean BMC BMC changes across the disuse period were generally consistent, with the exception of the non-bed rest ULLS study. The mean absolute bone loss was systematically greater in BMCt than in BMCc or BMCsub, but also showed much more variation during disuse (Fig. 3 and Table 5). The mean absolute EIBL rates indicated a trend toward accelerated trabecular bone loss after $\sim 60$ days of disuse (of note, according to the LTBR data only). No such trend was observed in the cortical compartment (see Fig. 3 and Table 5). In contrast, the mean relative EIBLs were similar in all bone compartments (Table 5).

The multiple-regression analysis confirmed a positive relationship between EIBL and duration of disuse for both the cortical compartment in absolute $\left(R^{2}=0.42 ; P=0.024\right)$ and relative $\left(R^{2}=0.40 ; P=0.034\right)$ values, and the trabecular compartment in absolute $\left(R^{2}=0.38 ; P=0.045\right)$ and relative

\section{Table 4. Short-term error}

\begin{tabular}{lccc}
\hline \hline \multicolumn{1}{c}{ Study } & BMCc & BMCsub & BMCt \\
\hline LTBR & $0.7 \%$ & $1.3 \%$ & $0.4 \%$ \\
BBR & $0.8 \%$ & $0.9 \%$ & $0.6 \%$ \\
Valdoltra & $1.5 \%$ & $1.1 \%$ & $0.7 \%$ \\
ULLS & $1.8 \%$ & $0.9 \%$ & $0.8 \%$ \\
\hline
\end{tabular}


Immobilization period

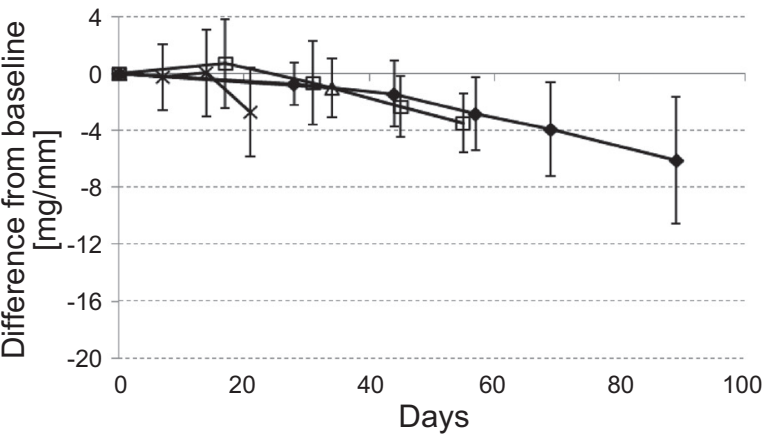

BMCc
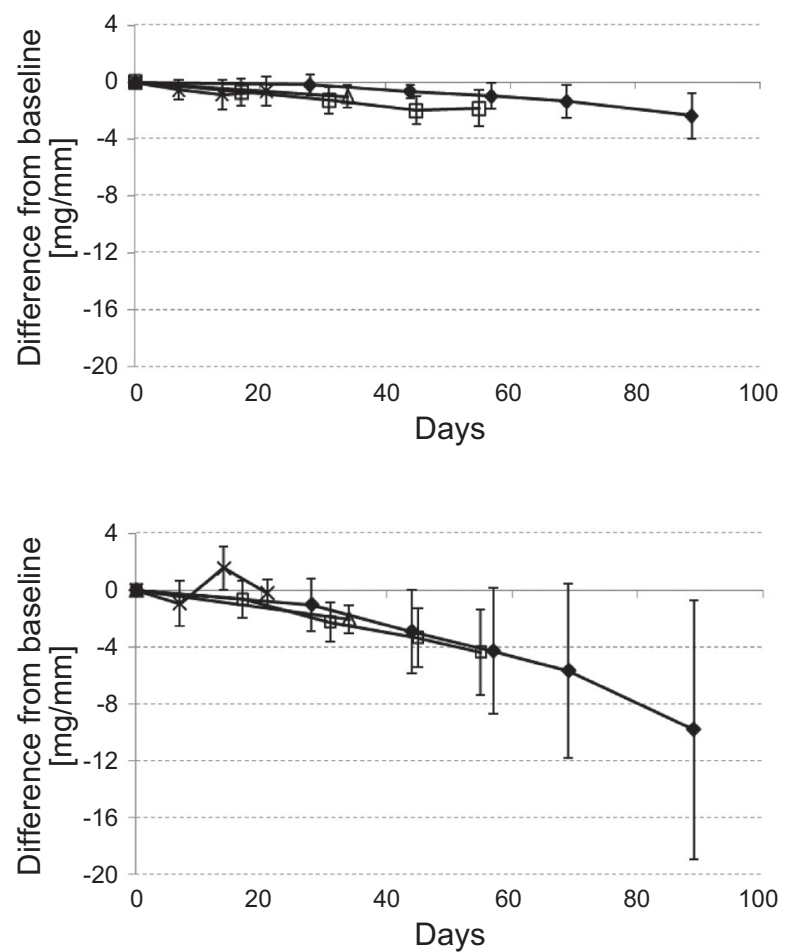

Recovery period
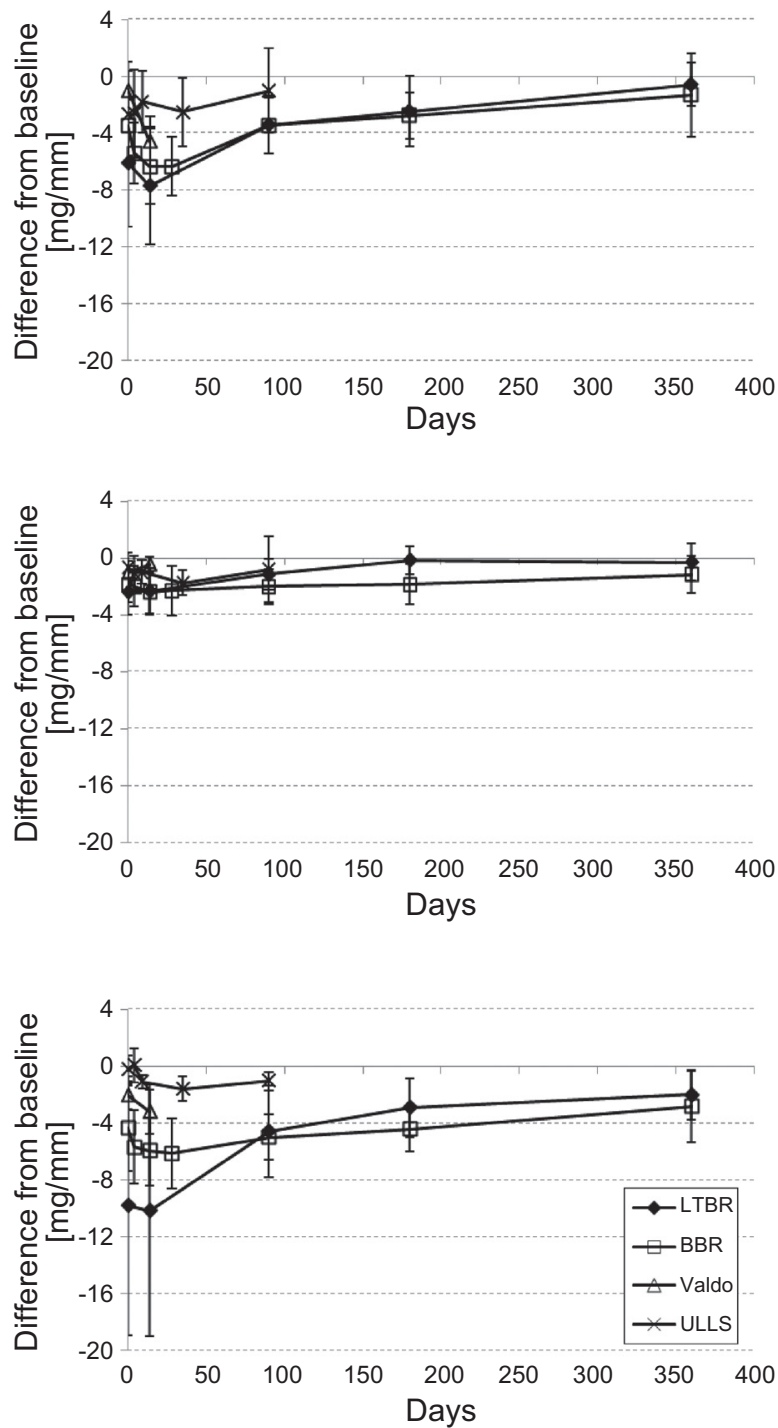

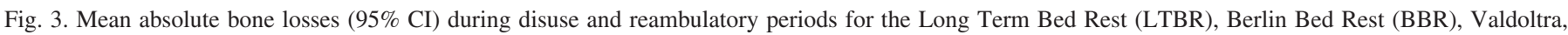

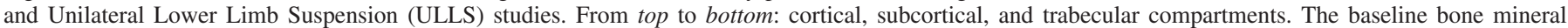

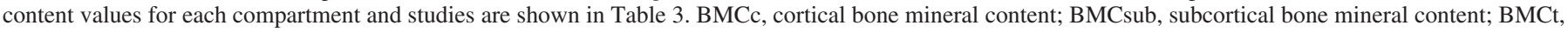
trabecular bone mineral content.

$\left(R^{2}=0.37 ; P=0.046\right)$ values. No association, however, was found between EIBL and baseline BMC values $(P>0.4)$.

The goodness of fit of the exponential model (Equation 4 ) during the disuse period, given in $R^{2}$ values, was $0.80,0.61$, and 0.87 ( $b$ values were $-0.00054,-0.00056$, and -0.00055$)$ for cortical, subcortical, and trabecular compartments, respectively. The simple quadratic and linear models (Equation 2 and Equation 3) did not improve prediction of bone loss in the subcortical compartment $\left(R^{2}=0.62\right.$ and 0.61 ; parameters, $a=$ $0.00006, b=0.0293$, and $b=-0.0258$, and $c=0.6112 ; P>$ 0.12 ), but these models were better descriptors of bone loss in the cortical $\left(R^{2}=0.94, a=-0.0007, b=-0.0071 ; R^{2}=\right.$ $0.97, b=-0.092, c=2.14)$ and trabecular compartments $\left(R^{2}=0.99, a=-0.0009, b=-0.0264 ; R^{2}=0.95, b=\right.$ $-0.12, c=2.11$ ), and clearly outperformed, within the given range of data, the exponential model $(P<0.005)$. The linear model better described changes in the cortical compartment
$(P=0.003)$, whereas the quadratic model better described changes in the trabecular compartment $(P=0.004)$.

Bone recovery patterns during reambulation. Absolute and relative recovery of $\mathrm{BMCc}, \mathrm{BMCsub}$, and $\mathrm{BMCt}$ at the distal tibia during the reambulation period is shown in Figs. 3 and 4, respectively. In general, mean BMC in all bone compartments returned virtually to baseline during reambulation, excluding the consistent $\sim 2 \mathrm{mg} / \mathrm{mm}$ trabecular loss in the longest LTBR and BBR studies. It is notable that the bone loss seemed to continue during the first approximately 15-30 days after termination of disuse in all bed rest studies. At the onset of reambulation, a systematically greater PRABL rate was observed in BMCc than in BMCt $(P=0.002$ in terms of absolute changes, and $P<0.0001$ in terms of relative changes), the non-bed rest ULLS study excluded, whereas the PRABL rate was marginal in BMCsub. Of interest, as shown in Table 5, the highest PRABL rate in the cortical compartment was in the shortest 
Immobilization period

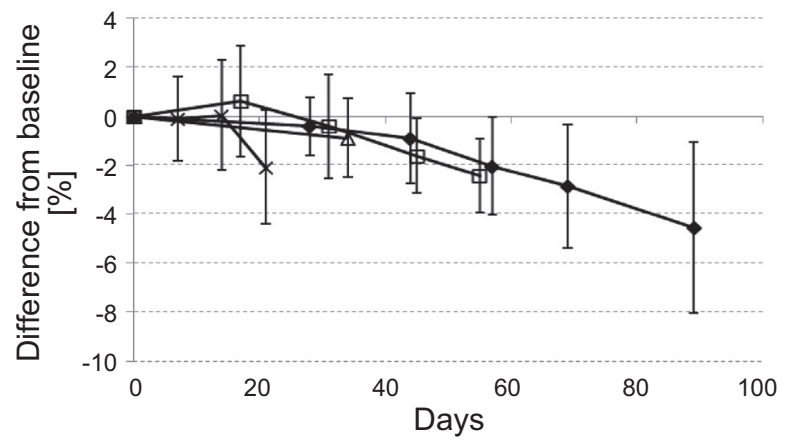

BMCc
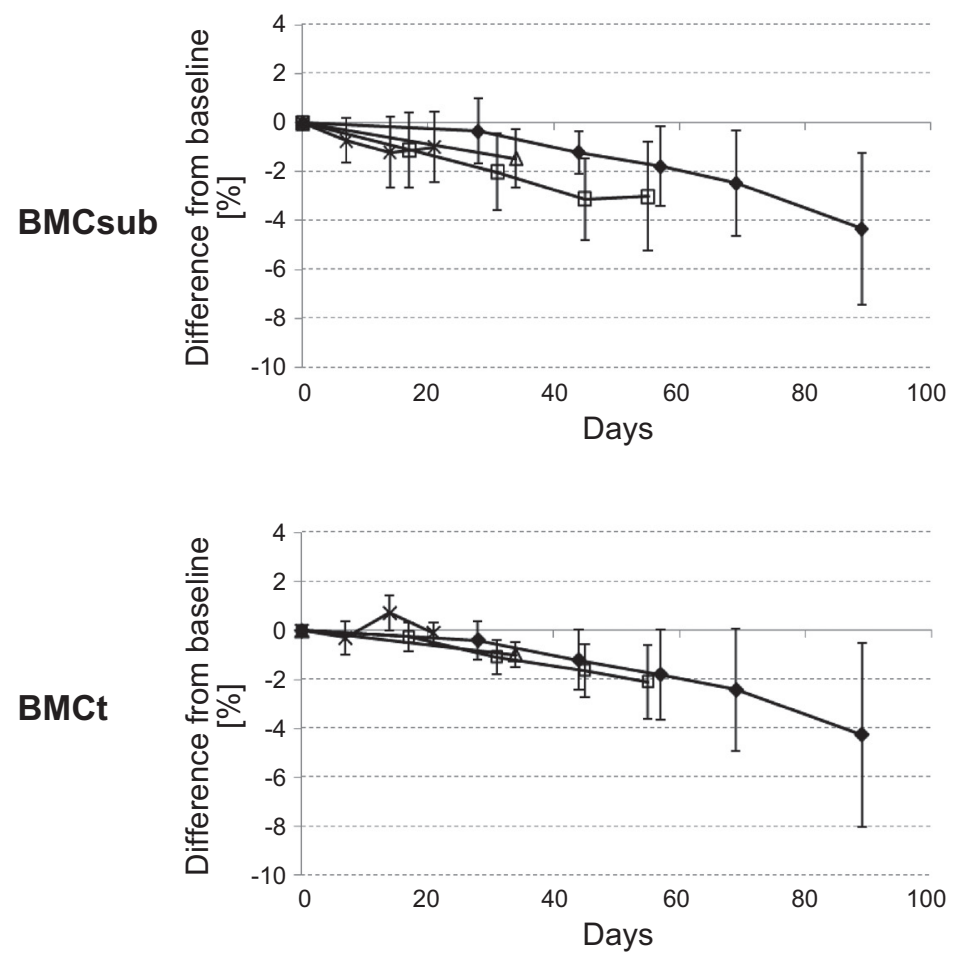

Recovery period
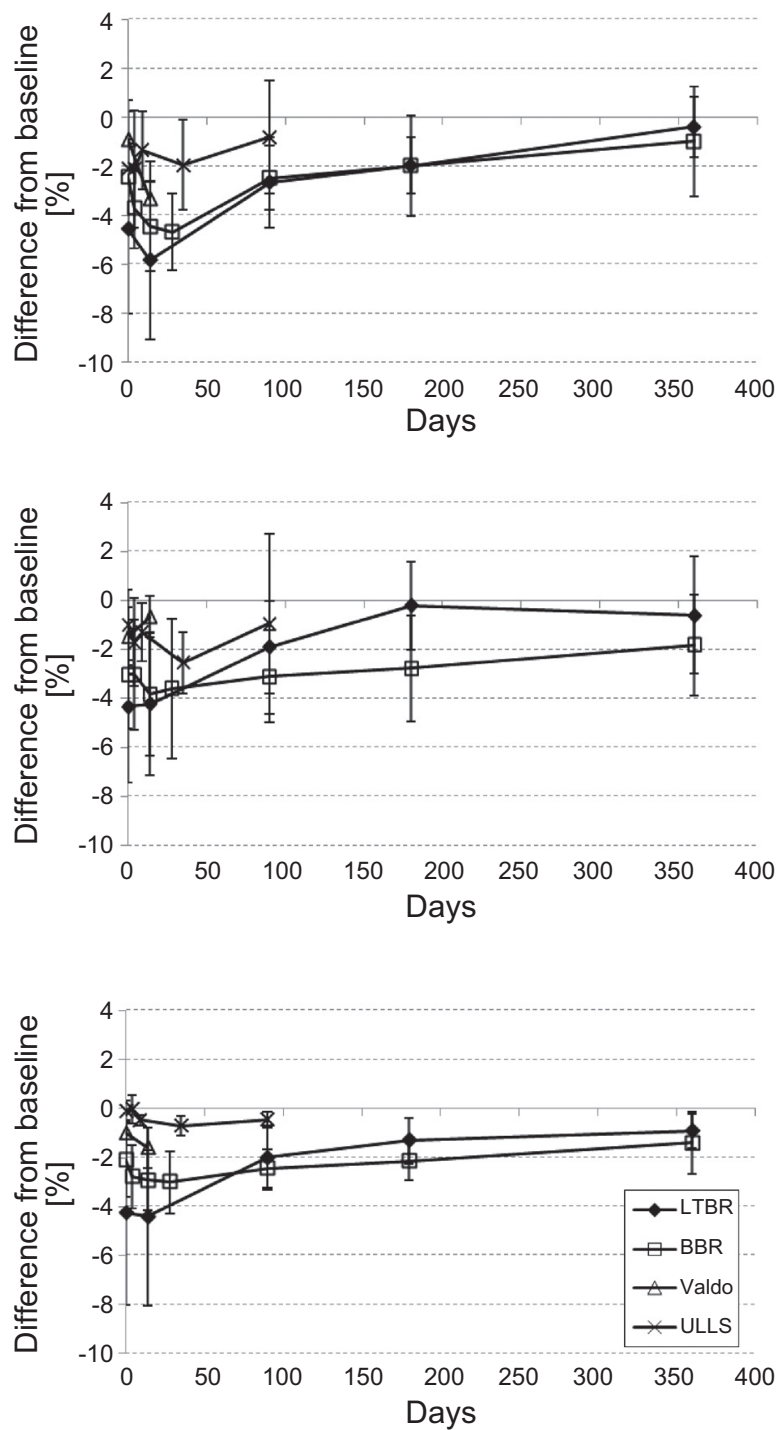

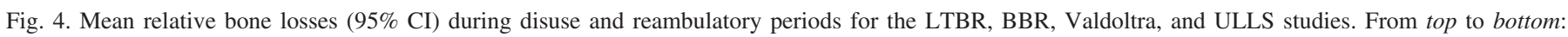
cortical, subcortical, and trabecular compartments. The baseline bone mineral content values for each compartment and studies are shown in Table 3 .

Valdoltra study, and was significantly higher than in the longest LTBR study $(P=0.034)$. Differences in PRABL rates in the trabecular compartment between these studies were not significant $(P>0.29)$. The mean absolute $\mathrm{PRABL}_{\max }$ seemed to be larger in the cortical than in the trabecular compartment for all studies except for the longest LTBR study (Table 5); however, none of the absolute differences between the cortical and trabecular compartments reached statistical significance $(P=0.46)$. In contrast, the relative mean $\mathrm{PRABL}_{\max }$ was systematically greater $(P=$ 0.048 ) in the cortical compartment than in the trabecular compartment.

The multiple-regression analysis gave support to the view that the PRABL rate in the cortical compartment would be negatively related to the duration of disuse $(P<0.05)$ and EIBL rate $(P<0.0007)$, and positively related to $\mathrm{PRABL}_{\max }$ $(P<0.028)$ both in absolute $\left(R^{2}=0.70 ; P=0.0006\right)$ and relative $\left(R^{2}=0.67 ; P=0.0016\right)$ terms. Similarly for the trabecular PRABL rate, $\mathrm{PRABL}_{\max }$ showed positive $(P<$
$0.03)$ and the EIBL rate showed negative $(P<0.003)$ association. These factors appeared to be good descriptors of the PRABL rate both in absolute $\left(R^{2}=0.59 ; P=0.013\right)$ and relative $\left(R^{2}=0.60 ; P=0.01\right)$ changes in trabecular BMC, whereas the duration of disuse showed no association $(P>0.25)$.

Bone gains during recovery were well explained by the exponential model (Equation 4$)$ in the cortical $\left(R^{2}=0.93, b=\right.$ $\left.0.0047 ; R^{2}=0.96, b=0.0073\right)$, subcortical $\left(R^{2}=0.97, b=\right.$ $\left.0.0019 ; R^{2}=0.92, b=0.0091\right)$, and trabecular $\left(R^{2}=0.96\right.$, $\left.b=0.0019 ; R^{2}=0.93, b=0.0068\right)$ compartments, respectively.

\section{DISCUSSION}

The main purpose of the present work was to reanalyze the pQCT data pooled from several disuse studies with a robust method to obtain a larger study cohort to investigate whether bone loss is different in cortical and trabecular bone compart- 
Table 5. Mean absolute and relative values, with 95\% CI, of maximal bone losses and bone loss rates induced during the end of immobilization and after the reambulation period

\begin{tabular}{|c|c|c|c|c|c|c|c|}
\hline & Compartment & Study & Duration & EIBL & $\mathrm{PRABL}_{\max }$ & EIBL-rate & PRABL-rate \\
\hline \multirow[t]{8}{*}{ Absolute values } & \multirow[t]{4}{*}{ Cortical } & LTBR & 90 & $-6.1 \pm 4.5$ & $-7.7 \pm 4.1$ & $-0.11 \pm 0.08$ & $-0.11 \pm 0.08$ \\
\hline & & BBR & 56 & $-3.5 \pm 2.1$ & $-6.3 \pm 2.7$ & $-0.12 \pm 0.18$ & $-0.19 \pm 0.10$ \\
\hline & & Valdoltra & 35 & $-1.0 \pm 2.1$ & $-4.6 \pm 1.7$ & $-0.03 \pm 0.06$ & $-0.24 \pm 0.10$ \\
\hline & & ULLS & 24 & $-2.7 \pm 3.1$ & $-2.5 \pm 2.4$ & $-0.39 \pm 0.34$ & $0.005 \pm 0.04$ \\
\hline & \multirow[t]{4}{*}{ Trabecular } & LTBR & 90 & $-9.8 \pm 9.1$ & $-10.1 \pm 8.9$ & $-0.21 \pm 0.16$ & $-0.02 \pm 0.06$ \\
\hline & & BBR & 56 & $-4.4 \pm 3.0$ & $-6.0 \pm 2.4$ & $-0.10 \pm 0.15$ & $-0.11 \pm 0.08$ \\
\hline & & Valdoltra & 35 & $-2.0 \pm 1.0$ & $-3.2 \pm 1.6$ & $-0.06 \pm 0.03$ & $-0.08 \pm 0.07$ \\
\hline & & ULLS & 24 & $-0.2 \pm 1.0$ & $-1.6 \pm 0.9$ & $-0.25 \pm 0.20$ & $-0.03 \pm 0.03$ \\
\hline \multirow[t]{8}{*}{ Relative values } & \multirow[t]{4}{*}{ Cortical } & LTBR & 90 & $-4.6 \pm 3.5$ & $-5.8 \pm 3.2$ & $-0.08 \pm 0.06$ & $-0.08 \pm 0.07$ \\
\hline & & BBR & 56 & $-2.4 \pm 1.5$ & $-4.4 \pm 1.8$ & $-0.08 \pm 0.14$ & $-0.13 \pm 0.07$ \\
\hline & & Valdoltra & 35 & $-0.9 \pm 1.6$ & $-3.3 \pm 1.6$ & $-0.03 \pm 0.05$ & $-0.16 \pm 0.06$ \\
\hline & & ULLS & 24 & $-2.1 \pm 2.3$ & $-1.9 \pm 1.8$ & $-0.12 \pm 0.09$ & $-0.01 \pm 0.01$ \\
\hline & \multirow[t]{4}{*}{ Trabecular } & LTBR & 90 & $-4.3 \pm 3.7$ & $-4.4 \pm 3.6$ & $-0.09 \pm 0.07$ & $-0.01 \pm 0.03$ \\
\hline & & BBR & 56 & $-2.1 \pm 1.5$ & $-2.9 \pm 1.2$ & $-0.05 \pm 0.07$ & $-0.05 \pm 0.04$ \\
\hline & & Valdoltra & 35 & $-1.0 \pm 0.5$ & $-1.6 \pm 0.8$ & $-0.03 \pm 0.02$ & $-0.04 \pm 0.04$ \\
\hline & & ULLS & 24 & $-0.1 \pm 0.5$ & $-0.7 \pm 0.4$ & $-0.12 \pm 0.09$ & $-0.01 \pm 0.01$ \\
\hline
\end{tabular}

Maximal bone losses are shown as $\mathrm{mg} / \mathrm{mm}$ and $\%$; bone loss rates are shown as $\mathrm{mg} \cdot \mathrm{mm}^{-1}$.day ${ }^{-1}$ and $\% /$ day. EIBL, bone loss at the end of immobilization

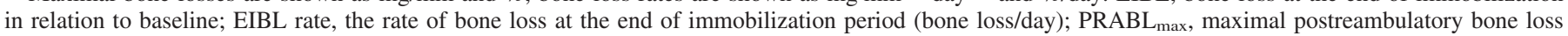
in relation to EIBL; PRABL rate, postreambulatory bone loss rate (bone loss/day).

ments, as previous small studies had separately indicated (13, $21,22,32$ ), and whether the bone responses to disuse and subsequent reambulation were modulated by the different designs of the studies.

To achieve this, the present work uniquely focused on consistent image processing that permitted analysis of the same bone cross-sectional areas throughout the entire follow-up periods so that changes in bone compartments were monitored and analyzed in a more comparable fashion. Our results indicate that during the disuse period, mean absolute bone losses were most pronounced in the trabecular compartment (Fig. 3). Of interest, mean absolute bone losses in the cortical compartment increased during a short period (2-3 wk) after reambulation, the longest LTBR study excluded. During this immediate postambulation period, the total absolute cortical bone loss in disuse studies shorter than 60 days (about 2 mo) exceeded the absolute trabecular bone losses induced during the preceding disuse period. This is in line with recent findings from immobilization studies $(1,18,20,22)$. In the longer 90-day LTBR study, however, the total mean absolute cortical bone loss did not exceed the total mean trabecular bone loss induced during disuse. This was despite faster cortical bone deterioration immediately after reambulation, and mean trabecular bone loss remained greater than cortical bone loss.

Study design. The specific nature of the disuse design and the body position in particular [0 degrees in the Valdoltra study (22) and in the BBR study (1), and -6 degrees head tilted down in the LTBR study (21)] seemed not to substantially modulate bone loss. The mean bone loss in all bone compartments in all bed rest studies were concordant during the disuse period and showed similar patterns during the reambulation period as well. Clearly, the magnitude of disuse-induced mean bone loss is related to the total duration of intervention $(4,18$, $20-22,27)$, as was also confirmed by the present regression analysis. It should be noted that interindividual variability seemed to increase with disuse duration.

Apparently, the seminal LTBR study had a direct impact on the design of subsequent bed rest studies. The shorter 35-day Valdoltra study was performed to corroborate the findings of the LTBR study (21) and findings from a long-duration space flight (13) suggesting that the majority of bone loss occurred primarily in the cortical compartment. Thus the duration of successive bed rest studies was limited to a maximum of 60 days. This was considered to be a sufficient duration for the prediction of future bone loss (18). The ULLS study, in turn, was considered a feasible alternative to more complicated and expensive bed rest study designs that might cause not only extensive physical effects but also psychological effects (23). However, the ULLS study, with a completely different mode of disuse and younger participants, showed different patterns in bone responses to disuse compared with bed rest studies (Figs. 3 and 4).

Specifically, the mean BMCt in the ULLS study showed an unexpected increase during the first days of disuse. This increase could be related to an increase in venous pressure that partially could have prevented the trabecular bone loss, as suggested by a rat study by Bergula et al. (5). However, a similar increase was also reported in a trabecular BMD in a short, 21-day, -6 degrees head-tilted-down bed rest study (29), but not in the bed rest studies assessed similarly in the present work. That the majority of bone loss in the ULLS study was predominantly cortical is concordant with findings from the bed rest studies $(1,18,22)$. On the other hand, the short duration (24 days) and poor quality of image data compromised this analysis. Longer limb suspension studies are needed to more appropriately assess whether the bone loss patterns would agree with the bed rest studies or have a unique pattern. At present, it seems that unilateral suspension is not a feasible model for bed rest studies.

Patterns of disuse induced bone losses. In the assessed bed rest studies, trabecular bone loss showed a short delay $(\sim 17$ days) at the onset of disuse. This delay is in line with a recent finding from the bed rest study of Wang et al. (33), in which they reported only marginal trabecular bone loss at the distal tibia during the first 30 days of disuse. After this initial quiescent period, trabecular bone loss increased rather linearly until the $\sim 60$ th day of disuse. With longer disuse, mean trabecular bone loss seemed to gradually increase and showed 
more substantial loss than occurred in the cortical bone compartment. However, the number of participants who underwent long disuse (duration $>60$ days) remained small because only one bed rest study lasted that long. In general, substantial trabecular bone loss is a well known consequence of bone disuse (27) or a long-duration space flight mission (32). Nevertheless, previous findings from bed rest studies have suggested that the most pronounced bone loss would be in the cortical compartment $(3,18,21,22)$. It may be that bone losses from the cortical and trabecular compartments have different time courses such that cortical loss starts almost immediately and is mostly linear over time, whereas trabecular loss is initially delayed but then becomes aggravated with longer immobilization. The ultimate question is which factors would underlie the apparently different dynamics in response to an altered loading environment?

Patterns of postreambulation bone loss. In agreement with the previously reported disuse studies $(1,4,18,21-23)$, the current analysis showed that bone deterioration continues during the first days after reambulation and culminates between the 15th and 30th days after reambulation. Furthermore, bone loss in all compartments is almost completely restored ( $99 \%$ on average) within $1 \mathrm{yr}$ after termination of disuse. The immediate postreambulation cortical losses excluded, no study-specific modulations were observed during the later reambulation period. However, the greater postreambulatory bone-loss rate within the cortical compartment compared with the trabecular compartment seems to be related to maximal postreambulatory bone loss and negatively to the rate of bone loss at the end of immobilization. This relation may indicate a hysteresis or lag in skeletal adaption by which the hysteresis is attributable to the delays in the differentiation of the osteoblasts while the osteoclasts remain active. Nevertheless, further studies are needed to better understand the cellular-level processes behind cortical bone loss, because only a small part of the variation could be explained.

Strengths and limitations. The strengths of the present analysis are 1) the evaluation of pooled data from four out of the seven so-far-published disuse interventions performed with pQCT, and 2) completely harmonized bone analysis by applying the new threshold-free image processing and segmentation method known to enhance pQCT image analysis $(7,9,17)$. The method allowed accurate detection of the cortical bone without the use of simple thresholds that can substantially modulate pQCT results $(12,34)$. A direct comparison of disuse studies was not previously possible because each study used different threshold settings for bone analysis $(18,21-23)$.

Some limitations warrant further discussion. Basically, even the use of an advanced image processing technique cannot overcome inherent limitations caused by the coarse spatial resolution of pQCT images, as resolution limits the performance of any image processing, the threshold-based analyses in particular (31). In addition, despite having the largest ever pooled and carefully analyzed data sets from several disuse studies, the total sample size still remained small. This is even more pronounced at later time points of the disuse or recovery period when data from only a limited number of subjects and studies were available. The small sample size limited the statistical power of the analysis to detect potential, subtle differences in time-specific skeletal responses to disuse. This might have affected the bone loss models. Thus the possibility that the linear and quadratic models would not accurately represent the actual adaptation processes in bones at the later time points cannot be entirely ruled out. Furthermore, when considering the significance of the magnitude of bone loss, one must take into account the short-term error of this analysis approach (Table 4) and precision of pQCT-measured BMC values at the distal tibia $[<1.5 \%$ (25)]. Therefore, given the relatively small differences observed in BMC values and the sensitivity of pQCT, the presented results should be interpreted with caution.

Conclusion. Keeping inherent limitations of this analysis in mind, the present pooled analysis provided new and profound insights into patterns of compartment-specific bone losses during disuse and subsequent recovery. First, the specific design of the bed rest study seemed not to substantially influence bone loss and recovery patterns. Second, the largest mean absolute bone losses seemed to take place in the cortical compartment, but apparently only during the first 2 mo $(<60$ days). Third, as indicated by the longer LTBR study, continued disuse would result in the largest mean absolute bone loss in the trabecular compartment. Obviously, more long-duration studies are needed to confirm this. Because of the short duration and young subject ages of the ULLS study, the present analysis could not establish whether the unilateral suspension could be considered a feasible model for expensive and challenging bed rest study designs. Keeping the inherent statistical limitations of small disuse studies in mind, the present analysis showed that the use of robust image processing applied to the same subject-specific bone cross-sectional areas throughout the longitudinal studies enhanced bone analysis and revealed specific skeletal responses that would have remained concealed in conventional analysis. In addition, our analysis approach, if applied to future longitudinal studies, would allow direct interstudy comparisons. Obviously, more studies are needed to prove this concept.

\section{ACKNOWLEDGMENTS}

We thank all personnel of the participating institutions and coworkers for their advice and assistance during the disuse studies. We are grateful to the study participants. This work would not have been possible without their selfless contribution.

\section{GRANTS}

Support for this study was provided by the Finnish Cultural Foundation, the Finnish Funding Agency for Technology and Inovations (Tekes), Project $718 / 31 / 2011$, and the Graduate School of Tampere University of Technology to T. Cervinka. Support was also provided by Competitive Research Funding of Tampere University Hospital Grant 9K121. Support for our participation in the LTBR study was supported by Institute Aerospace Medicine (DLR in Hamburg) Grant 50-WB 0156. The ULLS study was funded by European Space Agency MESM2 Project Grant 15097/01/NL/SH-CCN3. This study was also funded in part by the Osteoporosis and Muscle Atrophy Group of the Italian Space Agency, the Slovene Ministry of Defence, and the Gösta Fraenckel Foundation for Medical Research.

\section{DISCLOSURES}

No conflicts of interest, financial or otherwise, are declared by the author(s).

\section{AUTHOR CONTRIBUTIONS}

Author contributions: T.C., H.S., and J.R. conception and design of research; T.C. analyzed data; T.C. prepared figures; T.C. drafted manuscript; T.C., H.S., J.H., and J.R. edited and revised manuscript; T.C., H.S., J.H., and J.R. approved final version of manuscript; H.S., J.H., and J.R. interpreted results of experiments; J.R. performed experiments. 


\section{REFERENCES}

1. Armbrecht G, Belavý DL, Gast U, Bongrazio M, Touby F, Beller G, Roth HJ, Perschel FH, Rittweger J, Felsenberg D. Resistive vibration exercise attenuates bone and muscle atrophy in 56 days of bed rest: biochemical markers of bone metabolism. Osteoporos Int 21: 597-607, 2010.

2. Ashe MC, Khan KM, Kontulainen SA, Guy P, Liu D, Beck TJ, McKay HA. Accuracy of pQCT for evaluating the aged human radius: an ashing, histomorphometry and failure load investigation. Osteoporos Int 17: 1241-1251, 2006.

3. Belavý DL, Beller G, Armbrecht G, Perschel FH, Fitzner R, Bock O, Börst H, Degner C, Gast U, Felsenberg D. Evidence for an additional effect of whole-body vibration above resistive exercise alone in preventing bone loss during prolonged bed rest. Osteoporos Int 22: 1581-1591, 2011.

4. Beller G, Belavý DL, Sun L, Armbrecht G, Alexandre C, Felsenberg D. WISE-2005: bed-rest induced changes in bone mineral density in women during 60 days simulated microgravity. Bone 49: 858-866, 2011.

5. Bergula AP, Huang W, Frangos JA. Femoral vein ligation increases bone mass in the hindlimb suspended rat. Bone 24: 171-177, 1999.

6. Bolotin HH, Sievanen H. Inaccuracies inherent in dual-energy X-ray absorptiometry in in vivo bone mineral density can seriously mislead diagnostic/prognostic interpretations of patient-specific fragility. $J$ Bone Miner Res 16: 799-805, 2001.

7. Cervinka T, Hyttinen J, Sievänen $\mathbf{H}$. Enhanced bone structural analysis through pQCT image preprocessing. Med Eng Phys 32: 398-406, 2010.

8. Cervinka T, Hyttinen J, Sievänen $\mathbf{H}$. Threshold-free automatic detection of cortical bone geometry by peripheral quantitative computed tomography. J Clin Densitom 15: 413-421, 2012.

9. Cervinka T, Rittweger J, Hyttinen J, Felsenberg D, Sievänen H. Anatomical sector analysis of load-bearing tibial bone structure during 90-day bed rest and 1-year recovery. Clin Physiol Funct Imaging 31: 249-257, 2011.

10. Fyhrie DP, Schaffler MB. The adaptation of bone apparent density to applied load. J Biomech 28: 135-146, 1995.

11. Hangartner TN, Short DF. Accurate quantification of width and density of bone structures by computed tomography. Med Phys 34: 3777-3784, 2007

12. Kontulainen S, Liu D, Manske S, Jamieson M, Sievänen H, McKay H. Analyzing cortical bone cross-sectional geometry by peripheral QCT: comparison with bone histomorphometry. J Clin Densitom 10: 86-92, 2007.

13. Lang TF, Leblanc AD, Evans HJ, Lu Y. Adaptation of the proximal femur to skeletal reloading after long-duration spaceflight. $J$ Bone Miner Res 21: 1224-1230, 2006.

14. LeBlanc AD, Driscol TB, Shackelford LC, Evans HJ, Rianon NJ, Smith SM, Feeback DL, Lai D. Alendronate as an effective countermeasure to disuse induced bone loss. J Musculoskelet Neuronal Interact 2: 335-343, 2002.

15. Pavy-Le Traon A, Heer M, Narici MV, Rittweger J, Vernikos J. From space to Earth: advances in human physiology from 20 years of bed rest studies (1986-2006). Eur J Appl Physiol 101: 143-194, 2007.

16. Pieper S, Lorensen W, Schroeder W, Kikinis R. The NA-MIC kit: ITK, VTK, pipelines, grids and 3D slicer as an open platform for the medical image computing community. Proceedings of the 3rd IEEE International Symposium on Biomedical Imaging: From Nano to Macro. 2006, pp. 698-701.

17. Rantalainen T, Nikander R, Heinonen A, Cervinka T, Sievänen H, Daly RM. Differential effects of exercise on tibial shaft marrow density in young female athletes. J Clin Endocrinol Metab 98: 2037-2044, 2013.

18. Rittweger J, Beller G, Armbrecht G, Mulder E, Buehring B, Gast U, Dimeo F, Schubert H, de Haan A, Stegeman DF, Schiessl H, Felsenberg $D$. Prevention of bone loss during 56 days of strict bed rest by side-alternating resistive vibration exercise. Bone 46: 137-147, 2010.
19. Rittweger J, Beller G, Ehrig J, Jung C, Koch U, Ramolla J, Schmidt F, Newitt D, Majumdar S, Schiessl H, Felsenberg D. Bone-muscle strength indices for the human lower leg. Bone 27: 319-326, 2000.

20. Rittweger J, Felsenberg D. Recovery of muscle atrophy and bone loss from 90 days bed rest: results from a one-year follow-up. Bone 44: 214-224, 2009.

21. Rittweger J, Frost HM, Schiessl H, Ohshima H, Alkner B, Tesch P, Felsenberg D. Muscle atrophy and bone loss after 90 days' bed rest and the effects of flywheel resistive exercise and pamidronate: results from the LTBR study. Bone 36: 1019-1029, 2005.

22. Rittweger J, Simunic B, Bilancio G, De Santo NG, Cirillo M, Biolo G, Pisot R, Eiken O, Mekjavic IB, Narici M. Bone loss in the lower leg during 35 days of bed rest is predominantly from the cortical compartment. Bone 44: 612-618, 2009.

23. Rittweger J, Winwood K, Seynnes O, de Boer M, Wilks D, Lea R, Rennie M, Narici M. Bone loss from the human distal tibia epiphysis during 24 days of unilateral lower limb suspension. J Physiol 577, Pt 1: 331337, 2006.

24. Shackelford LC, LeBlanc AD, Driscoll TB, Evans HJ, Rianon NJ, Smith SM, Spector E, Feeback DL, Lai D. Resistance exercise as a countermeasure to disuse-induced bone loss. J Appl Physiol 97: 119-129, 2004.

25. Sievänen H, Koskue V, Rauhio A, Kannus P, Heinonen A, Vuori I. Peripheral quantitative computed tomography in human long bones: evaluation of in vitro and in vivo precision. J Bone Miner Res 13: 871-882, 1998.

26. Sievänen H. A physical model for dual-energy X-ray absorptiometryderived bone mineral density. Invest Radiol 35: 325-330, 2000

27. Sievänen H. Immobilization and bone structure in humans. Arch Biochem Biophys 503: 146-152, 2010.

28. Smith SM, Zwart SR, Heer M, Lee SM, Baecker N, Meuche S, Macias BR, Shackelford LC, Schneider S, Hargens AR. WISE-2005: supine treadmill exercise within lower body negative pressure and flywheel resistive exercise as a countermeasure to bed rest-induced bone loss in women during 60-day simulated microgravity. Bone 42: 572-581, 2008.

29. Smith SM, Zwart SR, Heer MA, Baecker N, Evans HJ, Feiveson AH, Shackelford LC, Leblanc AD. Effects of artificial gravity during bed rest on bone metabolism in humans. J Appl Physiol 107: 47-53, 2009.

30. Uebelhart D, Bernard J, Hartmann DJ, Moro L, Roth M, Uebelhart B, Rehailia M, Mauco G, Schmitt DA, Alexandre C, Vico L. Modifications of bone and connective tissue after orthostatic bedrest. Osteoporos Int 11: 59-67, 2000.

31. Veitch SW, Findlay SC, Ingle BM, Ibbotson CJ, Barrington A, Hamer AJ, Eastell R. Accuracy and precision of peripheral quantitative computed tomography measurements at the tibial metaphysis. J Clin Densitom 7: 209-217, 2004.

32. Vico L, Collet P, Guignandon A, Lafage-Proust MH, Thomas T, Rehaillia M, Alexandre C. Effects of long-term microgravity exposure on cancellous and cortical weight-bearing bones of cosmonauts. Lancet 355: 1607-1611, 2000.

33. Wang H, Wan Y, Tam KF, Ling S, Bai Y, Deng Y, Liu Y, Zhang H, Cheung WH, Qin L, Cheng JC, Leung KS, Li Y. Resistive vibration exercise retards bone loss in weight-bearing skeletons during 60 days bed rest. Osteoporos Int 23: 2169-2178, 2012.

34. Ward KA, Adams JE, Hangartner TN. Recommendations for thresholds for cortical bone geometry and density measurement by peripheral quantitative computed tomography. Calcif Tissue Int 77: 275-280, 2005.

35. Zerwekh JE, Ruml LA, Gottschalk F, Pak CY. The effects of twelve weeks of bed rest on bone histology, biochemical markers of bone turnover, and calcium homeostasis in eleven normal subjects. $J$ Bone Miner Res 13: 1594-1601, 1998

36. Zwart SR, Hargens AR, Lee SM, Macias BR, Watenpaugh DE, Tse K, Smith SM. Lower body negative pressure treadmill exercise as a countermeasure for bed rest-induced bone loss in female identical twins. Bone 40: 529-537, 2007. 